\title{
CONDITION ASSESSMENT OF POWER CABLES IN THE ENEL DISTRIBUTION NETWORK, CONSIDERATIONS AFTER 2 YEARS TRIAL
}

\author{
G. Rizzi*, M. Manzo**, C. Sidoti ** \\ *CESI **ENEL Distribuzione \\ Italy \\ *grizzi@cesi.it
}

\section{INTRODUCTION}

Cable MV lines represent a very sensitive portion in the distribution network, because of the potential technical and economical impact of failures. In the last decade several diagnostic systems were developed aiming at assessing the cable line conditions mainly trough the use of a diagnostic indicator based on the PD (Partial Discharge) activity. Several reports are available in the technical literature about their performances and their ability to spot out potential defects; however the analysis is often based on a quite limited set of case studies. On the other hand, ENEL Distribuzione carried out a very extensive survey of its distribution cable network and the present paper reports and discusses some of the major results both in terms of analysis of the technical validity of the diagnostic systems used and of the economical benefit obtainable with this activity

\section{CABLE CONDITION ASSESSMENT ACTIVITY CARRIED OUT}

ENEL Distribuzione outsourced to three different companies (CESI was one of the contractors) in the years 2002-2003 a large campaign aimed at assessing the condition of an outstanding part of its medium voltage cable network. The condition assessment was based on PD measurement with the off line techniques; each contractor used its own system and its own methods and criteria to process the results. The drivers, the approach and the technical requirements of the campaign were presented in [1]; only preliminary results were reported being the activity in progress; the main results achieved are reported and analysed in the present paper.

Figure 1 reports the number of sections tested and the cumulated line length (the length of the tested cable being computed multiplying by a factor three, to take the three phases into account, the length of the line) subdivided in the Italian macroregion.

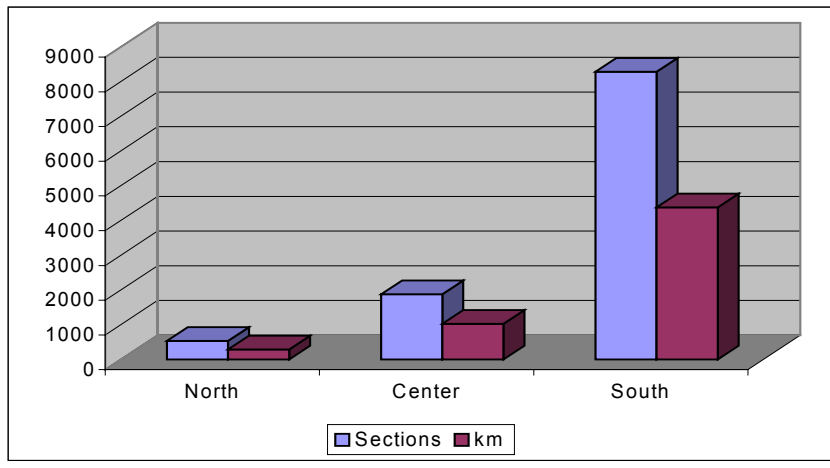

Figure 1: number of tested sections and relevant cumulated length

Most of the tests were carried out in South Italy where the failure rate was higher.

The critical points were ranked, as described in [1], in four levels; for every cable section classified at level 4 or 3 a repetition of the measurement after 5 and 3 years respectively was advised, while for levels 2 and 1 a repair action was indicated as necessary within 1 year and few month respectively.

Figure 2 reports the total number of critical points ranked 1 and 2 for the three macro-regions. The figure shows a very wide spread of the number of critical points among the regions with the highest number in Southern Italy; rationalizing the results on the base of $100 \mathrm{~km}$ of line, the results are reported in Figure 3.

Figure 3 also shows a very wide spread. In fact, in spite of the limited numbers of section tested, the number of critical points $1 \& 2$ found for Central Italy is much higher than that relevant to the other two regions; this fact could not be related to a worse condition of the cable, but only to an optimised selection of the sections to be tested

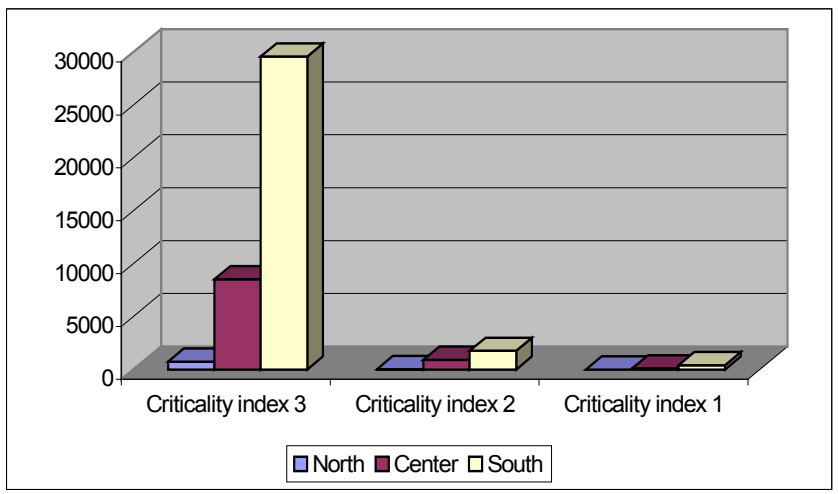

Figure 2: number of critical point subdivided for levels 


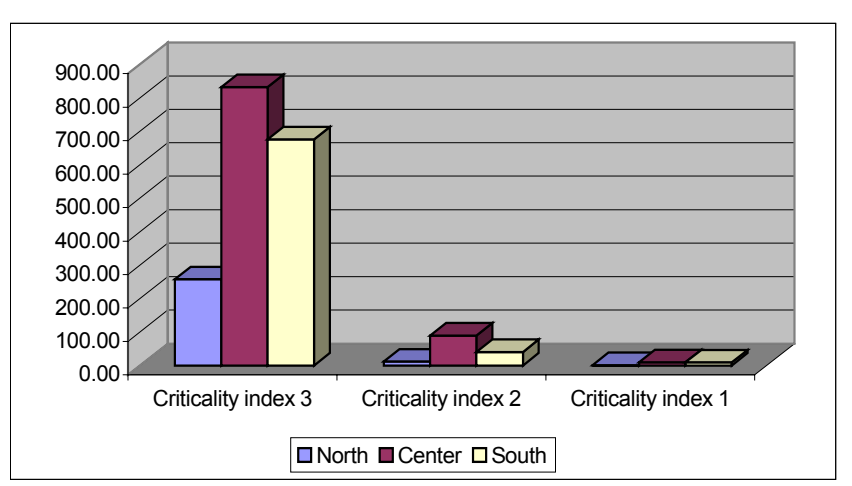

Figure 3: number of critical points rationalised on $100 \mathrm{~km}$ of line

For northern and southern Italy the results obtained are in agreement with the failure rates, reported in Figure 4.

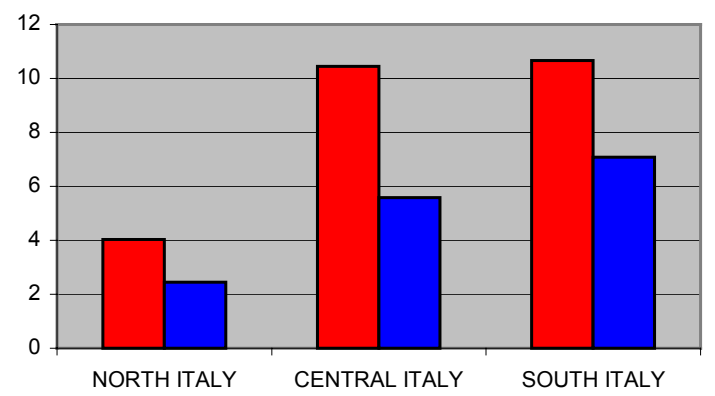

Figure 4: Failure rate (red overall, blue electrical)

\section{Determination of the physical position of defected parts}

In case of the assessment of cable conditions with criticality indexes 1 or 2, i.e. needing immediate corrective action, the physical determination of the defected point was carried out on the terrain. This activity is very important when the exact map of the cable line path is not available. When the cable lines run in cities the determination of the defected point is not easy because of the presence of other services as for example gas pipes, water pipes, low voltage networks and so on- In spite of this difficulties, in $95 \%$ of the cases the component indicated by diagnostic measurement was found when digging. The activity was carried out injecting a suitable signal in the cable line removed from service; typically, in a working day the physical position of the defected points belonging to three sections were carried out.

\section{Analysis of the removed part}

The critical points ranked $1 \& 2$ were removed and analyzed jointly by ENEL Distribuzione and the contractor's experts to verify the presence of any reason justifying the removal of the cable portions. The analysis were performed on the base of visual inspection disassembling the removed components. The results of the inspection were ranked in 3 levels: regular, not regular and invalid.. Regular analyses are those where the reasons of PD activity was evident, while not regular analyses are those where no reasons for PD activity were found; finally, invalid analysis are those where the incorrect removal of the component does not allow the analysis itself. Moreover, it was decided not to proceed to any repair work on some specific components, even if the PD activity detected was very high; this decision was taken on the base of the experience gained: as an example a very high PD activity was observed on resin types terminations even if without any defect; moreover no corrective action was taken in case the defect appeared to be on the cable bulk, in consideration of the difficulty to localize on the terrain the exact defected point.

A list of situations leading to a regular/non regular analysis was prepared; Table 1 reports a list of the defects leading to a regular classification of the removed component.

Table 1: List of the defects leading to "regular" analysis

\begin{tabular}{|c|c|}
\hline Component & Defect \\
\hline $\begin{array}{c}\text { Joint/termination } \\
\text { (all type of } \\
\text { insulation) }\end{array}$ & $\begin{array}{l}\text { Wrong assembling sequence } \\
\square \text { Use of material not specified in } \\
\text { assembling instruction } \\
\square \text { Incorrect welding between earth strip } \\
\text { and screen of the cable } \\
\square \text { Moisture penetration } \\
\square \text { Defected material } \\
\square \text { Presence of bodies }\end{array}$ \\
\hline $\begin{array}{l}\text { Joint/termination } \\
\text { (impregnated } \\
\text { paper insulation) }\end{array}$ & $\begin{array}{l}\text { Paper incorrectly positioned (presence } \\
\text { of voids between paper layers) } \\
\square \text { Voids between insulating tube } \\
\square \text { Cut paper } \\
\square \text { Carbonized paper } \\
\square \text { Incorrect removal of semiconductive } \\
\text { layer } \\
\text { Wrong resin polymerization }\end{array}$ \\
\hline $\begin{array}{l}\text { Joint/termination } \\
\text { (impregnated } \\
\text { paper insulation) }\end{array}$ & $\begin{array}{l}\text { Change of color on limited part of the } \\
\text { insulating material } \\
\square \text { Farinage effect } \\
\text { Cut of primary insulation } \\
\text { Incorrect removal of semiconductive } \\
\text { layer }\end{array}$ \\
\hline
\end{tabular}

Some examples of the defects found are shown in Figure 5 to Figure 7. The internal inspection on removed parts evidenced non regular analysis in $18 \%$ of the cases. All the other cases can be consequently considered as avoided failures.

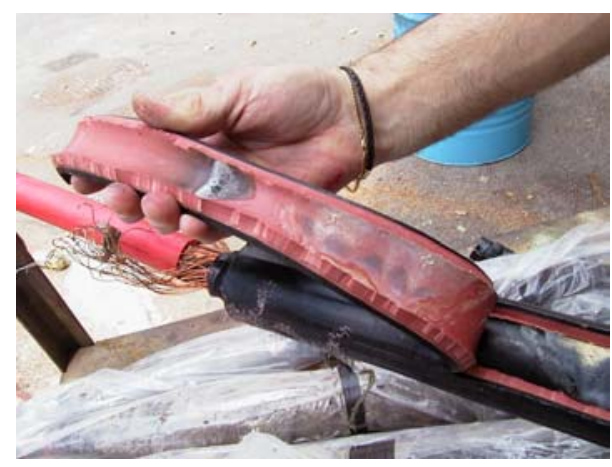

Figure 5: example of incorrect thermo-shrinking 


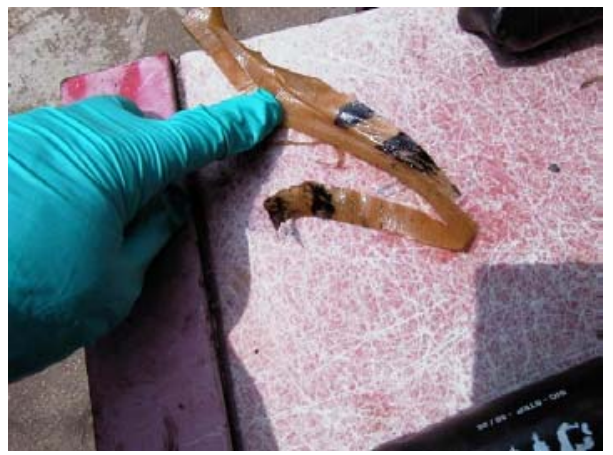

Figure 6: Example of carbonized paper

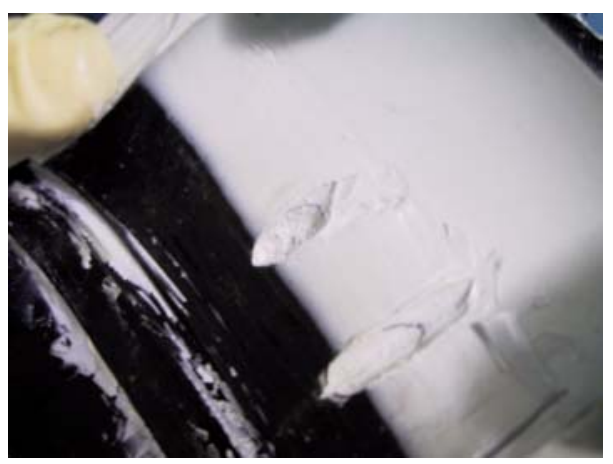

Figure 7: Example of defected material

\section{ECONOMICAL ASPECTS}

\section{Cost of the test}

The evaluation of the cost of the test considers all the components involved in the condition assessment of the cable line, and in particular:

$$
C_{t}=C_{s}+C_{u p}+C_{l}+C_{r}
$$

where $C_{s}$ is the cost of the measurement, $C_{u p}$ the cost of the utility personnel necessary to operate the network during the measurement, $C_{l}$ the cost for the physical location of the defected points and $C_{r}$ the cost for the repair.

\section{Cost of the avoided failure}

In order to evaluate the economical effectiveness of the condition assessment, the cost of avoided failure was taken into consideration: i.e. the total cost of the test reported to the number of real defective points found, that would have lead to a failure within a short period of time. The evaluation of the cost of each avoided failure was made according to the following formula:

$$
C_{a f}=\frac{C_{t}}{n_{a f}}=\frac{C_{t}}{n_{12} *\left(1-n_{n r}\right)}
$$

where:

口 $C_{a f}$ is the cost of each avoided failure

$C_{t}$ is the total cost for testing $100 \mathrm{~km}$ of cable lines
․ $n_{a f}$ is the number of avoided failures

- $\quad n_{12}$ is the number of defective points ranked (1 and 2: i.e. for which repair operations were advised)

- $n_{n r}$ is the fraction of visual inspections after the removal of the defected parts which were ranked as not regular.

Figure 8 reports the cost of avoided failure for each of the Italian macro-region; the figure shows a very wide spread in the costs, ranging from $4 \mathrm{k} €$ to $16 \mathrm{k} €$; this spread depends on the accuracy in the selection of the cable portions to be tested. We have seen in Figure 3 that for central Italy the number of the defected points ranked $1 \& 2$ was the highest: the selection of the cable portions to be tested was very effective (i.e. a large amount of cable portions selected for the tests by the local network manager on the base of its knowledge of the local network performances showed the presence of defects); this has reduced dramatically the cost of each avoided failure.

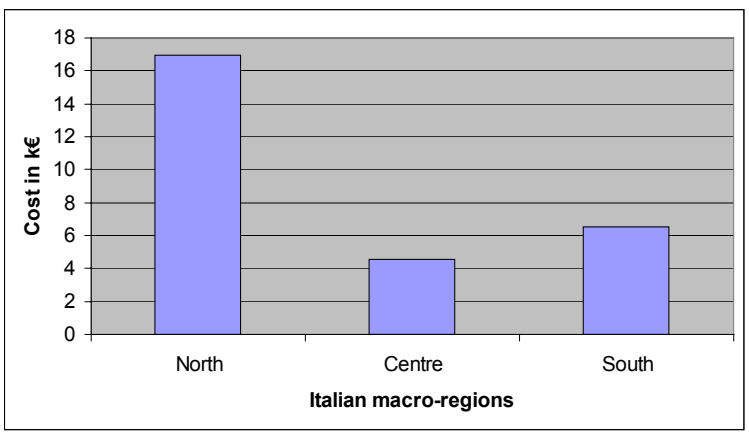

Figure 8: Cost in $k €$ for each avoided failure

The contribution of each component of the cost of test to the total cost of test was calculated for the three Italian macro-regions, see Figure 9.

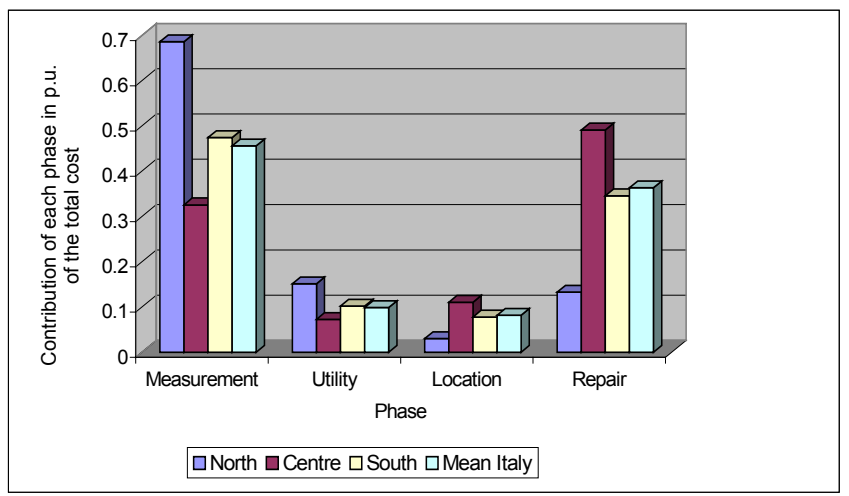

Figure 9: Contribution of the different sub phase on the overall cost for each avoided failure

The figure shows that measurement and repair costs account for about $80 \%$ of the overall cost, while the other two phases account for $10 \%$ each. In addition, the figure shows that the spread for each component can be very high, depending on the selection of the section to be tested; in fact, the higher the number of defected points ranked $1 \& 2$ the lower the cost of each avoided failure and the relative cost of the measurement. 
The presence of several operators able to carry out the condition assessment of power cables motivates the users of such services to ask for a reduction in the price of the service. The effects of the reduction of the price of the measurement (and of the corresponding cost if seen from the user point view) on the total cost of avoided failure is shown in Figure 10. The figure shows that the reduction in the overall cost is less than proportional; in fact, in the case of the central Italy a reduction of the measurement cost of $40 \%$ of the present cost would lead only to a $25 \%$ reduction in the overall cost. This fact is linked essentially to the nonnegligible contribution of the repair cost .

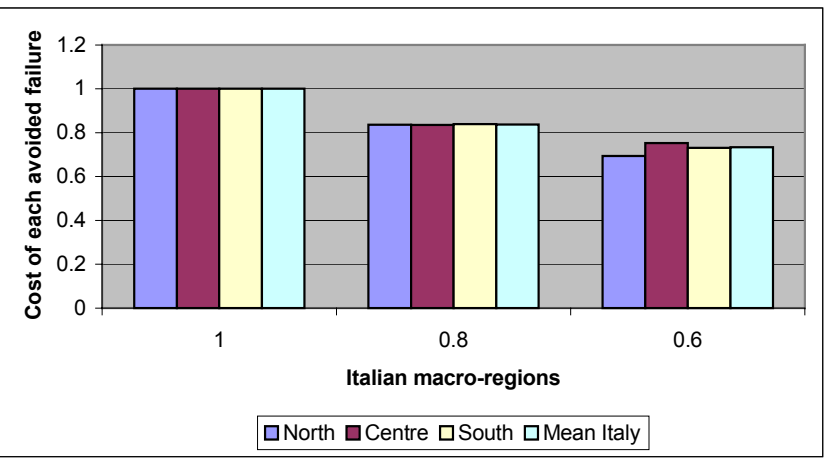

Figure 10: Overall cost of any avoided failure vs the reduction of measurement cost

\section{Failure cost}

The cost of each failure was calculated with the same approach used for the cost of the test, that is:

$$
C_{f}=C_{e}+C_{l}^{\prime}+C_{r}^{\prime}+P
$$

where:

$C_{f}$ is the cost of failure

$C_{e}$ is the cost of non-delivered energy during the failure (considered negligible in this approach)

a $C_{l}^{\prime}$ is the cost for the location of the failed point

a $C_{r}^{\prime}$ is the cost of the repair operations for the located fault

- $P$ is the cost derived by the application of the national rules on the quality of the service.

In Italy, the Authority for Electrical Energy and Gas (AEEG) has chosen as indicator of the quality of service the cumulated time per year of unavailability of energy (DCCE) for low voltage clients and the number of failure per yar for MV clients. Three areas were defined, namely: high, medium and low concentration, depending on the number of served client. For each geographical area of Italy AEEG has established a DCCE. The values are 25, 40 and 60 minutes per year for high, medium and low concentration, respectively. For a given area, DCCE is calculated as:

$$
D C C E=\frac{\sum_{L=1}^{N L A} D C C E_{\text {line }} * U_{i}}{\sum_{L=1}^{N L A} U l}
$$

where:

- $D C C E$ is the cumulated time of unavailability of energy

- $D C C E_{\text {line }}$ is the cumulated time of unavailability of energy relative to the line considered

- $U_{i}$ is the number of client belonging to the failed line

- $U l$ is the number of clients belonging to each line of the considered area

- $N L A$ are the lines belonging to the considered area In other words the time of unavailability is weighted for the total number of clients served in the considered area.

The target (LT) of the current year is defined on the base of the target of the previous year, the improvement required by AEEG and the limit defined for each area. If the DCCE, calculated at the end of the year using the real data, ranges between $\mathrm{LT} \pm 5 \%$ neither fine nor benefit shall be applied.

The simulation of the variation of the DCCE with the number of failure in a line is not simple because it is necessary to simulate the real lines belonging to given area and the management of the failure. The study case considers a MV line having the following characteristics:

a total length of the section $12.84 \mathrm{~km}$

口 number of $\mathrm{MV} / \mathrm{LV}$ transformer 30 ,

a number of LV clients 7750 ,

- number of remote controlled switches 3 ,

a number of manually operated switches 50

a power installed to the LV clients 18.9 MVA.

For that specific line we have simulated DCCE as a function of the number of failure per year; we notice that with 4 failures, the DCCE is equal to 26 minutes that is within the tolerance band of high concentration area. The next assumption was to consider this result applicable to an area having the cable length of $100 \mathrm{~km}$. Under this condition it is possible to derive from Figure 3 (considering that $80 \%$ of the post-diagnostics analysis showed regular results) the number of avoided failures. We have considered the data relevant to the North Italy region, being the data for that region the most conservative; the analysis brought up a value of 9.6 (i.e $12 * 0,8$ ). The simulation program was run to calculate DCCE under the condition that, in addition to the natural failure rate (4), the not fixed defected points will fail in 1, 2, 3, 4 and 5 years; the estimated DCCE values are reported in Table 2

Table 2: Number of failure per year and relevant DCCE

\begin{tabular}{|c|c|}
\hline $\begin{array}{c}\text { Failure per year } \\
\text { (number) }\end{array}$ & $\begin{array}{c}\text { DCCE } \\
\text { (minutes) }\end{array}$ \\
\hline 14.2 & 90 \\
\hline 9.1 & 58.1 \\
\hline 7.4 & 47.4 \\
\hline 6.6 & 42.4 \\
\hline 6 & 38.7 \\
\hline
\end{tabular}


The possible penalties/benefits are calculated with the formula given by AEEG, i.e:

$$
P=R E C \cdot\left[\left(P O T_{1} \cdot C_{1}\right)+\left(P O T_{2} \cdot C_{2}\right)\right]
$$

where:

REC is the difference between the target DCCE and the real DCCE, if it is within the tolerance $( \pm 5 \%)$ band equal 0

- $\mathrm{POT}_{1}$ energy sold to the non domestic clients divided by 8760 , for sake of simplicity we have considered the installed power

a $\mathrm{POT}_{2}$ energy sold to the domestic clients divided by 8760 , for sake of simplicity we have considered the installed power

- $\mathrm{C}_{1}$ and $\mathrm{C}_{2}$ are coefficient given by $\mathrm{AEEG}$ and reported in the following table

\begin{tabular}{|c|c|c|c|c|}
\hline $\begin{array}{c}\text { High } \\
\text { concentration } \\
\text { (minutes) }\end{array}$ & $\begin{array}{c}\text { Medium } \\
\text { concentration } \\
\text { (minutes) }\end{array}$ & $\begin{array}{c}\text { Low } \\
\text { concentration } \\
\text { (minutes) }\end{array}$ & $\begin{array}{c}\mathrm{C}_{1} \\
(€ / \mathrm{min} \\
/ \mathrm{kW})\end{array}$ & $\begin{array}{c}\mathrm{C}_{2} \\
(€ / \mathrm{min} \\
/ \mathrm{kW})\end{array}$ \\
\hline $\mathrm{DCCE} \leq 25$ & $\mathrm{DCCE} \leq 40$ & $\mathrm{DCCE} \leq 60$ & 0,024 & 0,012 \\
\hline $25<\mathrm{DCCE} \leq 75$ & $40<\mathrm{DCCE} \leq 120$ & $60<\mathrm{DCCE} \leq 7180$ & 0,036 & 0,018 \\
\hline $75 \leq \mathrm{DCCE}$ & $120 \leq \mathrm{DCCE}$ & $180 \leq \mathrm{DCCE}$ & 0,048 & 0,024 \\
\hline
\end{tabular}

When the result of the formula is positive there is a benefit to receive, on the contrary when it is negative there is a penalty to be paid.

\section{Determination of the advantages of the condition-based maintenance option}

A comparison between the condition based maintenance option (i.e. the application of the diagnostic tools discussed in this paper) and the corrective maintenance option (i.e. the run-to-failure and repair option) is carried out in the following.

The following table reports the different phases contributing to the overall cost in the two cases considered.

\begin{tabular}{|c|c|}
\hline $\begin{array}{c}\text { Condition Based Maintenance } \\
\text { (Diagnostic) }\end{array}$ & $\begin{array}{c}\text { Corrective Maintenance } \\
\text { (Run-to-failure) }\end{array}$ \\
\hline $\begin{array}{c}\text { Diagnostic measurement } \\
\text { (including the costs of the } \\
\text { Service and utility personnel) }\end{array}$ & Possible penalty \\
\hline Location of the defected point & Location of the failed point \\
\hline Repair & Repair \\
\hline
\end{tabular}

As first approximation we considered equivalent for the two options the costs for the failure location and repair. Figure 11 reports the economical evaluation, in case of LV Clients, for the considered line and for different Italian macro-regions; the evaluations were done considering the two cases of all residential Clients and half residential Clients. The bars represent the gain/loss for each failure, positive sign is for gain, while negative is for loss.

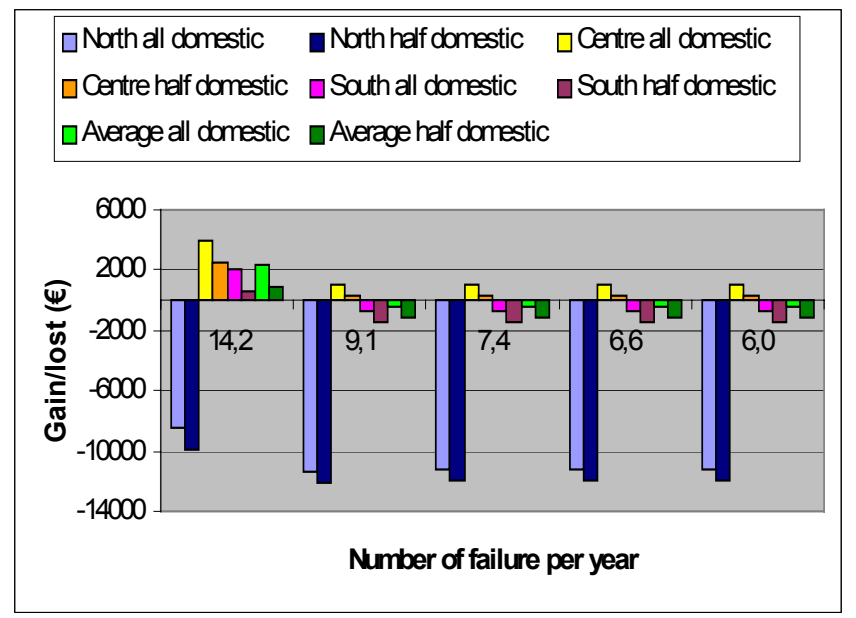

Figure 11: Gain/loss for diagnostic/waiting situations as a function of the number of failures per year

Figure 11 shows once more the importance of the accuracy in the selection of the sections to be tested: in fact, when the number of critical points detected during diagnostics tests is reduced the cost of the test is higher than the benefit obtained reducing DCCE; on the contrary, when the selection is very accurate there is a benefit even in case of only few extra failure avoided each year.

For MV Clients new rules will be applied starting from 2006 , in that case only the number of failures is to be taken into consideration, similarly for the $\mathrm{LV}$, the Clients are classified in the three areas (HC, MC, LC) and for each area a free number of failure is allowed and namely, 3, 4 and 5 for $\mathrm{HC}, \mathrm{MC}$ and LC respectively; the fine, calculated for each extra failure with a maximum of twice the free number, is proportional to the power delivered to the Clients as reported in Table 3 for $\mathrm{HC}$ area

Table 3: Fine for MV Clients

\begin{tabular}{|c|c|c|}
\hline $\begin{array}{c}\text { Power delivered } \\
(\mathrm{kW})\end{array}$ & $\begin{array}{c}\text { Fine/failure } \\
(€)\end{array}$ & $\begin{array}{c}\text { Max fine } \\
(€)\end{array}$ \\
\hline 100 & 175 & 525 \\
\hline 500 & 875 & 2650 \\
\hline 1000 & 1400 & 4200 \\
\hline
\end{tabular}

\section{Final considerations}

It has been demonstrated that one of the crucial points to have advantages in the application of this type of Condition Based Maintenance policy is the reduction of the measurements cost that can be achieved acting on different aspect: i.e. the productivity, the cost of the test and the accurate selection of the section to be tested. As this last aspect is concerned, Figure 8 has shown that the cost can be reduced dramatically.

The productivity obtained, during this extensive project, with the off line method can be considered quite high: in fact the test of one section (three cables) lasted for 2 hours and 40 minutes in average, all inclusive (operations to put the section out of service and grounded, and after the test again in service, the time for the joints position evaluation, the first processing of the obtained results and the time to 
move the van from one secondary substation to the following).

The calculation reported in Figure 11 were repeated assuming a productivity of 5 sections per day, that is, maintaining a global working time 10 hours, a time of only 2 hours for each section; the advantages is of the order of $1000 €$, improvement not sufficient to change basically the trend reported in Figure 11.

The other aspect considered is the flat reduction of the cost of the test, the calculation done considering the cost of the Service company equal to $70 \%$ of the present cost has shown that the loss are reduced of few thousand $€$ for the bad selection and that the improvement is few hundred $€$ in case of a good selection.

\section{An alternative approach to the off-line condition assessment: the on-line monitoring}

In the last years a new type of instrument was made available on the market, allowing the measurement of PD activity during service. Usually the sensor is a HFCT located around the ground connection of the cable; the most advanced can work without operator for long time, can send alarm signals and can locate the defected points under special network conditions. The experience gained in the last year with this kind of system has allowed to optimise the condition assessment procedure in order to maximise the ratio benefit/cost. The basic assumption is that being the sensors non invasive and having a cost quite limited they can be installed once and left there without the need of their removal. The installation, that normally shall be done with the secondary substation out of service, can be carried every time when the impact on the network asset is minimised. The installation time can be estimated in about 40 minutes per section (three phases). Once the sensors are installed the measuring instrument can be connected any time during the normal service. Under these conditions the estimated productivity is of 8 sections per day, 4 instruments provided that every cabin has at least 2 sections,

A reduced team of 2 people (1 for the Service Company and the other of the electrical Utility ) is sufficient instead of the 4 people necessary for out of service approach.

Figure 12 reports the economical calculations repeated for the same line in case of on-line measurement. The figures shows that only in cases of not carefully selection of the sections losses are still present even if of reduced amount, in all the other situation economical advantages exist even in case only two avoided failure per year.

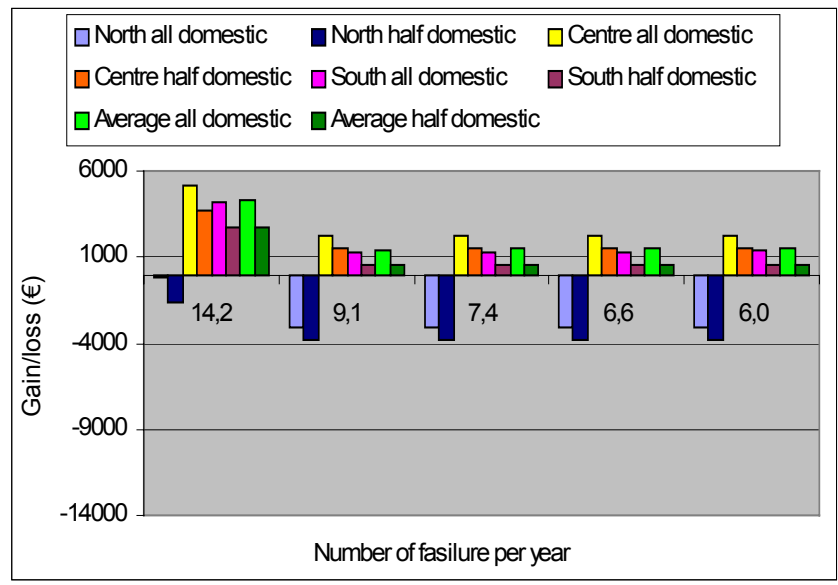

Figure 12: Economical evaluation in case of on line measurements

\section{Conclusions}

- The diagnostic tests, together with other actions, can be very effective in reducing the outages time in a cable network, identifying critical point before their failure.

- The data of the very large campaign, presented in first paragraphs, have shown that the technical results achieved are of good quality.

- In addition, the economical aspects were investigated to evaluate the correctness of the approach used; on the base of the cost of each avoided failure.

- As expected the higher is the convenience the better is selection of the sections to test, nevertheless in some cases the measurement cost are still too important with respect to the economical benefit gained reducing the overall number of failures.

a Considering the rules presently working in Italy, it was demonstrated that PD measurement under service conditions are very effective in improving the economical aspects.

\section{REFERENCES}

[1] M. de Nigris, G. Rizzi, E. Di Marino, G. Bonini Baldini, A. Fara, 2003,"Condition assessment of power cables in in the ENEL Distribuzione network", CIRED 2003 Barcelona 12-15 May, Session 1 paper 66 